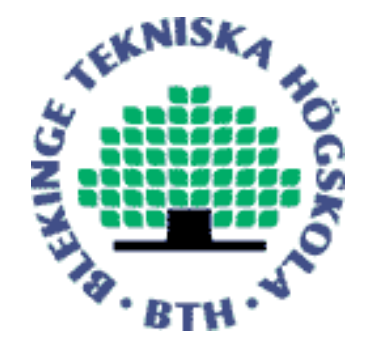

Copyright (C) 2014 IEEE.

Citation for the published paper:

MIMO Amplify-and-Forward Relay Systems with Dissimilar Channel Characteristics

Trung Q. Duong, Xianfu Lei, Hans-Jürgen Zepernick

International Conference on Computing, Management and Telecommunications

(ComManTel)

2014 Danang, Vietnam

This material is posted here with permission of the IEEE. Such permission of the IEEE does not in any way imply IEEE endorsement of any of BTH's products or services Internal or personal use of this material is permitted. However, permission to reprint/republish this material for advertising or promotional purposes or for creating new collective works for resale or redistribution must be obtained from the IEEE by sending a blank email message to pubs-permissions@iee.org.

By choosing to view this document, you agree to all provisions of the copyright laws protecting it. 


\title{
MIMO Amplify-and-Forward Relay Systems with Dissimilar Channel Characteristics
}

\author{
Trung Q. Duong*, Xianfu Lei ${ }^{\dagger}$, and Hans-Jürgen Zepernick ${ }^{\ddagger}$ \\ * Queen's University Belfast, Belfast, United Kingdom (e-mail: trung.q.duong@qub.ac.uk) \\ $\dagger$ Utah State University, Utah, USA (e-mail: xflei81@gmail.com) \\ $\ddagger$ Blekinge Institute of Technology, Karlskrona 37179, Sweden (e-mail: hans-jurgen.zepernick@bth.se)
}

\begin{abstract}
In this paper, we investigate the asymmetric property of multiple-input multiple-output (MIMO) dual-hop amplifyand-forward (AF) relay networks. We consider the difference of the two hops in terms of both fading channels and scattering environment. In particular, we analyze the symbol error probability (SEP) of a MIMO orthogonal space-time block code (OSTBC) AF relay network in which the first and second hop undergo Rayleigh fading with a rich-scattering environment and Nakagami- $m$ fading with a poor-scattering environment, respectively. Moreover, an asymptotic SEP expression yielding insights on the diversity gain is also obtained.
\end{abstract}

Index Terms-Amplify-and-forward (AF), relay networks, MIMO, OSTBC, Nakagami- $m$ fading.

\section{INTRODUCTION}

Most research on relay networks has assumed the propagation channel of dual-hop to be symmetric. However, in many practical scenarios, this assumption does not hold since the two hops can experience different fading conditions due to the mobility of mobile stations. For example, micro/macro cellular multi-hop transmissions have been characterized as a mixed Rayleigh/Rician propagation scenario in the WINNER II project [1]. Recently, several works have attempted to study the performance of amplify-and-forward (AF) relay networks taking into account the asymmetry of multi-hop transmission [2]-[6]. In particular, the symbol error probability (SEP) of a multiple-input multiple-output (MIMO) AF relay network with orthogonal space-time block code (OSTBC) transmission over Rayleigh/Rician fading channels has been studied in [2]. For the single antenna system, the performance of dual-hop AF relay networks in mixed Rayleigh/Rician or Rician/Rayleigh has been extensively investigated in [3]-[5]. Very recently, $\mathrm{Xu}$ et al. have derived closed-form expressions for outage probability and SEP of a mixed Nakagami- $m$ and Rician dualhop AF relay network [6].

Besides experiencing different fading channels, in practice, the two links, i.e., source-to-relay and relay-to-destination, can have different scattering environments. Souihli and Ohtsuki have studied different environments in the two hops by considering the first hop as a rich-scattering environment (i.e., the channel matrix is full rank) and the second hop as a poorscattering environment (i.e., the relay-to-destination link is a keyhole channel leading to the channel matrix possing a unit rank) [7], [8]. However, they only focused on the symmetric Rayleigh/Rayleigh fading channels in both hops. In addition, the performance of OSTBC transmissions with AF relaying over symmetric Rayleigh/Rayleigh fading channels has been addressed in [9]-[11].

To the best of the authors' knowledge, there is no previous work concerning both asymmetric property of the fading channel and different scattering environment for the two hops of relay networks. In this paper, therefore, the performance of MIMO AF relay networks with OSTBCs over mixed Rayleigh with rich-scattering and Nakagami- $m$ with poorscattering channels is studied. In particular, both closed-form and asymptotic expressions of SEP are presented. The diversity gain obtained for such a system is also provided.

\section{System And Channel Model}

We consider a downlink MIMO AF relay system where an $n_{0}$-antenna source $\mathrm{S}$ communicates with an $n_{2}$-antenna destination $\mathrm{D}$ through the help of an $n_{1}$-antenna fixed-gain AF relay $R$. Both $S$ and $R$ are fixed stations and can be deployed at strategic locations by the network operator, leading to the $\mathrm{S} \rightarrow \mathrm{R}$ link enjoying a rich-scattering environment, i.e., the channel matrix $\boldsymbol{H}_{1}$ is full rank. In contrast, D is a mobile station for a downlink scenario and it is reasonable to assume that its locations are subject to poor-scattering. With this assumption, the $\mathrm{R} \rightarrow \mathrm{D}$ link has a keyhole channel resulting in its corresponding channel matrix $\boldsymbol{H}_{2}$ having a rank deficiency, i.e., the rank of $\boldsymbol{H}_{2}$ is unit. Moreover, to account for the asymmetric fading among the two hops, we assume that the first hop follows Rayleigh fading with channel mean power $\Omega_{1}$ while the second hop is a Nakagami- $m$ fading channel with fading severity parameter $m$ and channel mean power $\Omega_{2}$.

In the first hop, $\mathrm{S}$ transmits an OSTBC matrix with rate $\mathcal{R}_{c}$ to $R$. In the second hop, the fixed-gain AF relay $R$ amplifies its received signal from $S$ and forwards it to $D$ in a semiblind mode. The channels in the two hops are assumed to be flat fading and perfectly known only to the receiver. Due to the orthogonal property of OSTBC, maximum-likelihood decoding can be converted to a symbol-wise decoding yielding the instantaneous signal-to-noise ratio (SNR) at D as follows [2]:

$$
\gamma_{\mathrm{D}}=\frac{\bar{\gamma}}{\mathcal{R}_{c} n_{0}}\left\|\left(\boldsymbol{I}_{n_{2}}+G_{A}^{2} \boldsymbol{H}_{2} \boldsymbol{H}_{2}^{\dagger}\right)^{-1 / 2} G_{A} \boldsymbol{H}_{2} \boldsymbol{H}_{1}\right\|_{\mathrm{F}}^{2}
$$

where $\|\cdot\|_{\mathrm{F}}$ denotes the Frobenius norm of a matrix, $G_{A}$ is the amplifying gain at $\mathrm{R}$ given as $G_{A}{ }^{2}=\left[n_{1}\left(\Omega_{1}+1 / \bar{\gamma}\right)\right]^{-1}$, and $\bar{\gamma}$ is the average SNR. 


\section{Symbol ERRor Probability ANAlysis}

For $M$-PSK modulation, the SEP of the considered system can be evaluated by the following integral:

$$
P_{e}=\frac{1}{\pi} \int_{0}^{\pi-\frac{\pi}{M}} \Phi_{\mathrm{D}}\left(\frac{g}{\sin ^{2} \theta}\right) d \theta
$$

where $g=\sin ^{2} \frac{\pi}{M}$ and $\Phi_{\mathrm{D}}(s)$ is the moment generating function (MGF) of $\gamma_{\mathrm{D}}$. The term $\Phi_{\mathrm{D}}(s)$ can be expressed as

$$
\begin{aligned}
& \Phi_{\mathrm{D}}(s)=\mathbb{E}_{\boldsymbol{H}_{2}}\left\{\operatorname { d e t } \left(\boldsymbol{I}_{n_{0} n_{1}}+s \frac{\bar{\gamma}}{\mathcal{R}_{c} n_{0}} \Omega_{1} G_{A}^{2} \boldsymbol{H}_{2}^{\dagger}\right.\right. \\
& \left.\left.\times\left(\boldsymbol{I}_{n_{2}}+G_{A}{ }^{2} \boldsymbol{H}_{2} \boldsymbol{H}_{2}^{\dagger}\right)^{-1} \boldsymbol{H}_{2} \otimes \boldsymbol{I}_{n_{0}}\right)^{-1}\right\} \\
& =\mathbb{E}_{\boldsymbol{H}_{2}}\left\{\frac{\operatorname{det}\left(\boldsymbol{I}_{n_{2}}+G_{A}^{2} \boldsymbol{H}_{2} \boldsymbol{H}_{2}^{\dagger}\right)}{\operatorname{det}\left[\boldsymbol{I}_{n_{2}}+G_{A}^{2}\left(1+\frac{s \bar{\gamma} \Omega_{1}}{\mathcal{R}_{c} n_{0}}\right) \boldsymbol{H}_{2} \boldsymbol{H}_{2}^{\dagger}\right]}\right\}^{n_{0}}
\end{aligned}
$$

where $\otimes$ denotes the Kronecker product. Let us denote $\lambda$ as the only non-zero eigenvalue of $\boldsymbol{H}_{2} \boldsymbol{H}_{2}^{\dagger}$. Since $\boldsymbol{H}_{2}$ is of unit rank, it is easy to see that $\lambda=\left\|\boldsymbol{H}_{2}\right\|_{\mathrm{F}}^{2}=X Y$, where $X$ and $Y$ are two gamma distributed random variables with shape parameters $N_{1}=m n_{1}$ and $N_{2}=m n_{2}$, respectively, and the same scale parameter $\Omega_{2} / m$. Using this property, we can rewrite (4) as

$$
\Phi_{\mathrm{D}}(s)=\int_{0}^{\infty} \frac{\lambda^{\frac{N_{1}+N_{2}}{2}-1}\left(1+G_{A}^{2} \lambda\right)^{n_{0}}}{\left[1+G_{A}^{2}(1+q s \bar{\gamma}) \lambda\right]^{n_{0}}} \mathcal{K}_{N_{2}-N_{1}}(2 \xi \sqrt{\lambda}) d \lambda
$$

where $\xi=\frac{m}{\sqrt{\Omega_{2}}} q=\frac{\Omega_{1}}{\mathcal{R}_{c} n_{0}}$, and $\eta=\frac{2 \xi^{N_{1}+N_{2}}}{\Gamma\left(N_{1}\right) \Gamma\left(N_{2}\right)}$. Further, $\mathcal{K}_{\nu}(\cdot)$ is the $\nu$ th-order modified Bessel function of the second kind [12]. To obtain a closed-form solution of $\Phi_{\mathrm{D}}(s)$, we express $\mathcal{K}_{N_{2}-N_{1}}(2 \xi \sqrt{\lambda})$ of (5) as

$$
\mathcal{K}_{N_{2}-N_{1}}(2 \xi \sqrt{\lambda})=\frac{1}{2} G_{02}^{20}\left(\left.\xi^{2} \lambda\right|_{\frac{N_{2}-N_{1}}{2}, \frac{N_{1}-N_{2}}{2}} ^{---}\right),
$$

where the result of [11, eq. (8.4.23.1)] is applied and $G(\cdot)$ is the Meijer's $G$-function [10, eq. (9.301)]. Applying (6) in (5) yields

$$
\begin{aligned}
\Phi_{\mathrm{D}}(s) & =\frac{\eta}{2(1+q s \bar{\gamma})^{n_{0}}} \int_{0}^{\infty} \frac{\lambda^{\frac{N_{1}+N_{2}}{2}-1}\left(1+G_{A}^{2} \lambda\right)^{n_{0}}}{\left[\lambda+\frac{1}{G_{A}^{2}(1+q s \bar{\gamma})}\right]^{n_{0}}} \\
& \times G_{02}^{20}\left(\left.\xi^{2} \lambda\right|_{\frac{N_{2}-N_{1}}{2}, \frac{N_{1}-N_{2}}{2}} ^{---} d \lambda\right. \\
& =\frac{\eta}{2(1+q s \bar{\gamma})^{n_{0}}} \sum_{n=0}^{n_{0}}\left({ }_{n}^{n_{0}}\right) G_{A}^{2 n-2 n_{0}} \int_{0}^{\infty} \frac{\lambda^{\frac{N_{1}+N_{2}}{2}+n-1}}{\left[\lambda+\frac{1}{G_{A}^{2}(1+q s \bar{\gamma})}\right]^{n_{0}}} \\
& \times G_{02}^{20}\left(\left.\xi^{2} \lambda\right|_{\frac{N_{2}-N_{1}}{2}, \frac{N_{1}-N_{2}}{2}} ^{--}\right) d \lambda,
\end{aligned}
$$

where the binomial expansion of $\left(1+G_{A}^{2} \lambda\right)^{n_{0}}$ is used in the last equality [12]. By applying the result of [11, eq. (2.24.2.4)], we can obtain the closed-form $\Phi_{\mathrm{D}}(s)$ as

$$
\begin{aligned}
& \Phi_{\mathrm{D}}(s)=\frac{\eta}{2 \Gamma\left(n_{0}\right)} G_{A}^{-\left(N_{1}+N_{2}\right)} \sum_{n=0}^{n_{0}}\left(\begin{array}{c}
n_{0} \\
n
\end{array}\right)(1+q s \bar{\gamma})^{-\frac{N_{1}+N_{2}}{2}-n} \\
& \times G_{1,3}^{3,1}\left(\left.\frac{\xi^{2}}{2 G_{A}^{2}(1+q s \bar{\gamma})}\right|_{n_{0}-n-\frac{N_{1}+N_{2}}{2}, \frac{N_{2}-N_{1}}{2}, \frac{N_{1}-N_{2}}{2}} ^{1-n-\frac{N_{1}+N_{2}}{2}}\right) .
\end{aligned}
$$

Substituting (9) into (2), we can obtain the numerical SEP of the considered system.
To gain further insight into the performance of the system, we turn to deriving the asymptotic SEP for high SNR. By making the variable change $\bar{\gamma} \lambda=x$, (5) can be rewritten as

$$
\begin{aligned}
\Phi_{\mathrm{D}}(s) & =\eta \bar{\gamma}^{-\frac{N_{1}+N_{2}}{2}} \int_{0}^{\infty}\left(\frac{1+G_{A}^{2} \bar{\gamma}^{-1} x}{1+G_{A}^{2}\left(\bar{\gamma}^{-1}+q s\right) x}\right)^{n_{0}} x^{\frac{N_{1}+N_{2}}{2}-1} \\
& \times \mathcal{K}_{N_{2}-N_{1}}\left(2 \xi \sqrt{\frac{x}{\bar{\gamma}}}\right) d x .
\end{aligned}
$$

To assess the SEP performance in the high SNR regime, we utilize the following tight approximation of the Bessel function $\mathcal{K}_{\nu}(x)$ for a small value of $x$ [12]:

$$
\mathcal{K}_{\nu}(x) \stackrel{(\operatorname{small} x)}{\approx}\left\{\begin{array}{cc}
\ln \left(\frac{1}{x}\right) & \text { for } \nu=0 \\
\frac{\Gamma(|\nu|)}{2}\left(\frac{2}{x}\right)^{|\nu|} & \text { for } \nu \neq 0
\end{array}\right.
$$

Accordingly, we differentiate the calculation of the asymptotic SEP for high SNR in two cases as follows:

- Case I: $N_{1} \neq N_{2}$. From (10) and (11), we can write the asymptotic $\Phi_{\mathrm{D}}(s)$ as

$$
\begin{aligned}
\Phi_{\mathrm{D}}(s) & \simeq \frac{\eta \Gamma\left(\left|N_{1}-N_{2}\right|\right)}{2} \bar{\gamma}^{-N_{\min }} \xi^{-\left|N_{1}-N_{2}\right|} \\
& \times \int_{0}^{\infty} \frac{x^{N_{\min }-1}}{\left(1+G_{A}^{2} q s x\right)^{n_{0}}} d x,
\end{aligned}
$$

where $N_{\min }=\min \left(N_{1}, N_{2}\right)$. When $n_{0} \geq N_{\min }+1$ holds, we can obtain the asymptotic SEP for $N_{1} \neq N_{2}$ as

$$
\begin{aligned}
\Phi_{\mathrm{D}}(s) & \simeq \frac{\eta \Gamma\left(\left|N_{1}-N_{2}\right|\right)}{2}\left(G_{A}^{2} q s \bar{\gamma}\right)^{-N_{\min }} \xi^{-\left|N_{1}-N_{2}\right|} \\
& \times L\left(N_{\min }, n_{0}\right),
\end{aligned}
$$

where

$$
L\left(k_{1}, k_{2}\right)=\sum_{k=0}^{k_{1}-1}\left(\begin{array}{l}
k_{1}-1 \\
k
\end{array}\right)(-1)^{k_{1}-k-1} \frac{1}{k_{2}-(k+1)} .
$$

Substituting (13) into (2) yields the asymptotic SEP for the case of $N_{1} \neq N_{2}$ as

$$
\begin{aligned}
P_{e} & \simeq \frac{\eta \Gamma\left(\left|N_{1}-N_{2}\right|\right)}{2 \pi}\left(G_{A}^{2} q g \bar{\gamma}\right)^{-N_{\min }} \xi^{-\left|N_{1}-N_{2}\right|} \\
& \times L\left(N_{\min }, n_{0}\right) c_{1},
\end{aligned}
$$

where $c_{1}=\int_{0}^{\pi-\frac{\pi}{M}} \sin ^{2 N_{\min }} \theta d \theta$. From the expression of the asymptotic SEP given in (18), we can find that the diversity order of the system is equivalent to $N_{\min }$ when $N_{1} \neq N_{2}$.

- Case II: $N_{1}=N_{2}$. In this case, we can write the asymptotic $\Phi_{\mathrm{D}}(s)$ from (10) and (11) as

$$
\begin{aligned}
\Phi_{\mathrm{D}}(s) & \simeq \eta \bar{\gamma}^{-N_{1}} \int_{0}^{\infty} \frac{x^{N_{1}-1}}{\left(1+G_{A}^{2} q s x\right)^{n_{0}}} \\
& \times\left[\ln \left(\sqrt{\frac{\bar{\gamma}}{4 \xi^{2}}}\right)-\frac{1}{2} \ln x\right] d x \\
& =\eta\left(G_{A}^{2} q s \bar{\gamma}\right)^{-N_{1}} L\left(N_{1}, n_{0}\right) \ln \left(\sqrt{\frac{\bar{\gamma}}{4 \xi^{2}}}\right) \\
& -\frac{\eta \bar{\gamma}^{-N_{1}}}{2} \int_{0}^{\infty} \frac{x^{N_{1}-1} \ln x}{\left(1+G_{A}^{2} q s x\right)^{n_{0}}} d x .
\end{aligned}
$$




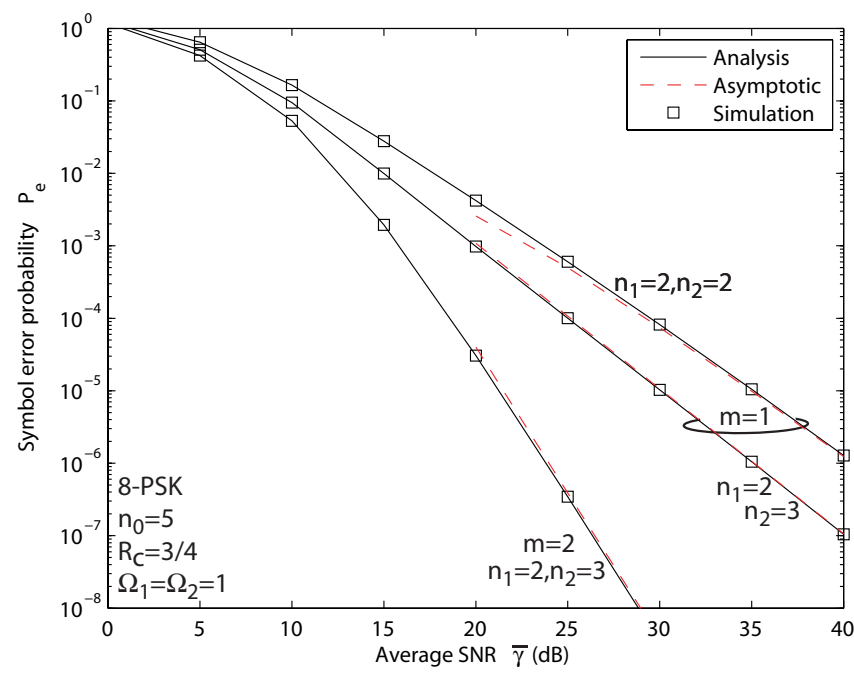

Fig. 1. SEP performance of a MIMO AF relay system for 8-PSK modulation versus average SNR.

When $n_{0} \geq N_{1}+1$ holds, we can obtain the asymptotic $\Phi_{\mathrm{D}}(s)$ for the case of $N_{1}=N_{2}$ as

$$
\begin{aligned}
& \Phi_{\mathrm{D}}(s)=\eta\left(G_{A}^{2} q s \bar{\gamma}\right)^{-N_{1}}\left[L\left(N_{1}, n_{0}\right) \ln \left(\sqrt{\frac{\bar{\gamma}}{4 \xi^{2}}}\right)-\right. \\
& \left.\frac{B\left(N_{1}, n_{0}-N_{1}\right)}{2}\left(\psi\left(N_{1}\right)-\psi\left(n_{0}-N_{1}\right)-\ln \left(G_{A}^{2} q s\right)\right)\right] .
\end{aligned}
$$

where $B(x, y)$ is the Beta function and $\psi(x)$ is the Euler Psi function [13]. Applying the asymptotic expression of $\Phi_{\mathrm{D}}(s)$ given by (18) into (2) yields the asymptotic SEP for the case of $N_{1}=N_{2}$ as

$$
P_{e}=\frac{\eta}{\pi}\left(G_{A}^{2} q g \bar{\gamma}\right)^{-N_{1}} c_{2},
$$

where $c_{2}=\int_{0}^{\pi-\frac{\pi}{M}}\left[L\left(N_{1}, n_{0}\right) \ln \left(\sqrt{\frac{\bar{\gamma}}{4 \xi^{2}}}\right)-\frac{B\left(N_{1}, n_{0}-N_{1}\right)}{2}\right.$ $\left.\times\left(\psi\left(N_{1}\right)-\psi\left(n_{0}-N_{1}\right)-\ln \left(G_{A}^{2} q g\right)+2 \ln (\sin \theta)\right)\right] \sin ^{2 N_{1}} \theta d \theta$. From the expression of the asymptotic SEP given in (19), it can seen that the diversity order of the system is equal to $N_{1}$ when $N_{1}=N_{2}$.

As $\min \left(N_{1}, N_{2}\right)=N_{1}$ when $N_{1}=N_{2}$, we can conclude from the above two cases that the diversity order of the considered system is equivalent to $\min \left(N_{1}, N_{2}\right)=m \min \left(n_{1}, n_{2}\right)$ when $n_{0} \geq m \min \left(n_{1}, n_{2}\right)+1$ holds. This indicates that the diversity order of the considered system increases with the Nakagami fading severity parameter $m$ and is limited by the minimum number of antennas at the relay and destination.

\section{NUMERICAL RESUlts}

To validate our analysis, we present some numerical and simulation results in this section. The average channel gains of the dual hops are both set to unity with $\Omega_{1}=\Omega_{2}=1$. The coding rate of OSTBC $R_{c}$ is set to $3 / 4$. We employ 8 -PSK modulation for the considered system, i.e. $M=8$.

Fig. 1 depicts the SEP of the MIMO AF relay system with 8 -PSK modulation versus average $\operatorname{SNR} \bar{\gamma}$, where $m=1,2$ and $n_{0}=5$. In other words, we consider the cases of Rayleigh/Rayleigh fading ( $m=1$ in the first and second hop) and Rayleigh/Nakagami-2 fading ( $m=1$ in the first hop and $m=2$ in the second hop). We consider two different antenna configurations of $n_{1}=2, n_{2}=2$ and $n_{1}=2, n_{2}=3$, corresponding to $N_{1}=N_{2}$ and $N_{1} \neq N_{2}$, respectively. As can be observed from Fig. 1, for different parameter settings, the analytical results match well with the simulations. Furthermore, the asymptotic results converge to the exact values in the high SNR region. In addition, it can be seen that the slope of the asymptotic curves is proportional to $m$. As such, the two system configurations of $n_{1}=2, n_{2}=2$ and $n_{1}=2, n_{2}=3$ exhibit the same diversity gain as expected.

\section{CONCLUSIONS}

This paper has investigated the performance of MIMO AF relaying under different channel characteristics for two hops. Assuming that the first hop experiences Rayleigh fading and rich scattering while the second hop experiences Nakagami$\mathrm{m}$ and poor scattering, exact and asymptotic expressions for the SEP have been derived. Our analytical results reveal significant insights into system performance in such severe channel conditions.

\section{REFERENCES}

[1] P. Kyösti, J. Meinilä, and L. Hentilä et al., WINNER II Interim Channel Models (IST-4-027756 WINNER II D1.1.1 V1.1) [Online]. Available: http://www.ist-winner.org/WINNER2-Deliverables/D1.1.1.pdf.

[2] T. Q. Duong, H. Shin, and E.-K. Hong, "Effect of line-of-sight on dualhop nonregenerative relay wireless communications," in Proc. IEEE Veh. Technol. Conf. (VTC-Fall), Baltimore, Maryland, Sep. 2007, pp. 571575.

[3] Y. Kim and H. Liu, "Infrastructure relay transmission with cooperative MIMO," IEEE Trans. Veh. Technol., vol. 57, no. 4, pp. 2180-2188, Jul. 2008.

[4] H. A. Suraweera, R. H. Y. Louie, Y. Li, G. K. Karagiannidis, and B. Vucetic, "Two hop amplify-and-forward transmission in mixed Rayleigh and Rician fading channels," IEEE Commun. Lett., vol. 13, no. 4, pp. 227-229, Apr. 2009.

[5] H. A. Suraweera, G. K. Karagiannidis, and P. J. Smith, "Performance analysis of the dual-hop asymmetric fading channel," IEEE Trans. Wireless Commun., vol. 8, no. 6, pp. 2783-2788, Jun. 2009.

[6] W. Xu, J. Zhang, and P. Zhang, "Performance analysis of dual-hop amplify-and-forward relay system in mixed Nakagami- $m$ and Rician fading channels," IET Electron. Lett., vol. 46, no. 17, pp. 1231-1232, Aug. 2010 .

[7] O. Souihli and T. Ohtsuki, "Cooperative diversity can mitigate keyhole effects in wireless MIMO systems," in Proc. IEEE Global Commununications Conf. (GLOBECOM), Hawaii, U.S.A., Nov/Dec 2009.

[8] - "The MIMO relay channel in the presence of keyhole effects," in Proc. IEEE International Commun. Conf. (ICC), Cape Town, South Africa, May 2010.

[9] T. Q. Duong, H. A. Suraweera, T. A. Tsiftsis, H.-J. Zepernick, and A. Nallanathan, "OSTBC transmission in MIMO AF relay systems with keyhole and spatial correlation effects," in Proc. IEEE Inter. Commun. Conf. (ICC), Kyoto, Japan, 2011, pp. 1-6.

[10] T. Q. Duong, H. A. Suraweera, C. Yuen, and H.-J. Zepernick, "Multikeyhole effect in MIMO AF relay downlink transmission with spacetime block codes," in Proc. IEEE Global Telecommun. Conf. (GLOBECOM), Houston, TX, Dec. 2011, pp. 1-5.

[11] T. Q. Duong, H. A. Suraweera, T. A. Tsiftsis, H.-J. Zepernick, and A. Nallanathan, "Keyhole effect in dual-hop MIMO AF relay transmission with space-time block codes," IEEE Trans. Commun., vol. 60, no. 12 , pp. 3683-3693, Dec. 2012.

[12] M. Abramowitz and I. A. Stegun, Handbook of mathematical functions. New York: Dover Publications Inc, 1974.

[13] I. S. Gradshteyn and I. M. Ryzhik, Table of Integrals, Series, and Products, 6th ed. San Diego, CA: Academic, 2000. 